\title{
RELATIONS ENTRE LES VARIATIONS PONDERALES DE LA TRUIE EN REPRODUCTION ET LES PERFORMANCES D'ÉLEVAGE
}

\author{
E. SALMON-LEGAGNEUR, C. LEgAULT, A. AUMAITRE
}

Station de Recherches sur l'Élevage des Porcs et Station centrale de Génétique animale, Centre national de Recherches zootechniques, 78 -Jouy-en-Josas

\section{SOMMAIRE}

Cent quatre-vingt-six truies de race Large White ayant produit 459 portées ont été utilisées dans cette étude. On a examiné d'une part les variations de poids (gains et pertes) au cours des portées successives ( $\mathrm{I}$ à 5 ), d'autre part les corrélations entre ces variations et les performances d'élevage (poids et nombre des porcelets aux différents stades de la lactation). On observe un certain parallélisme entre le gain de poids pendant la gestation, la perte pendant la lactation et le poids de la portée. Cette relation est discutée.

\section{INTRODUCTION}

Les variations de poids des truies, tant au cours de la gestation que de la lactation, sont en général importantes ( $\mathrm{I} 0$ à $50 \mathrm{p}$. Ioo du poids vif). Ce phénomène, bien connu des éleveurs, a été décrit ou étudié par plusieurs auteurs (I)ONALD et FLEMING, I938; ZELIER et al., I937; SCHAFER et GRANz, I955; LODGE et al., I96I; VANSchoubroek et EMbo, I962; MAJERCIAK, I962). Mais, si différents aspects physiologiques ont pu ainsi être précisés, le problème de la signification pratique et de l'intérêt économique de ces variations de poids se pose toujours. Doit-on considérer comme normal qu'une truie accumule, au cours de la gestation, des réserves importantes qui seront mobilisées au cours de la lactation, ou ce transfert théoriquement coûteux en énergie peut-il être évité par un rationnement mieux adapté aux besoins au cours de la lactation? Cet aspect nutritionnel a été souvent évoqué (SALMON-LEGAGNEUR, I965).

Sur un plan plus général, se trouve posé le problème des relations entre cer- 
taines caractéristiques maternelles (poids, gain de poids, conditions de parturition) et les résultats de la reproduction, gestation ou lactation.

C'est ce que nous avons voulu étudier à l'aide d'un nombre de données relativement important dont nous disposions.

\section{MATÉRIEL E'T MÉTHODË}

\section{Animaux}

Cent quatre-vingt-six truies de race Large White, ayant produit 459 portées ont été utilisées. Ces truies faisaient partie de l'élevage expérimental du C.N.R.Z. au cours de la période i954-196I. Les conditions d'exploitation sont décrites dans une autre publication (AumaírRe et al., rg66).

Ces truies ont été classées dans cette étude suivant leur numéro de portée. Or, par suite des éliminations naturelles dans tout élevage (mort, maladie, réforme), le nombre de portées effectuées n'est pas le même pour toutes les truies. En particulier, les portées de numéro supérieur à 5 sont moins nombreuses et ont dû être regroupées en une classe unique. Comme les animaux de ce groupe ont ainsi fait l'objet d'une certaine sélection (ce sont en général les meilleurs du troupeau), certaines conclusions doivent donc être interprétées avec réserve.

\section{Variables}

Celles-ci correspondent à un certain nombre de mesures simples effectuées sur chaque animal oul sa portée aux différentes étapes du cycle de reproduction (accouplement, parturition, lactation, serrage). Ces variables numérotées de I à 16 sont définies ci-dessous :

r. Durée de gestation : nombre de jours entre l'accouplement et la parturition.

2. Gain brut de gestation : différence entre le poids de la truie immédiatement avant parturition et à l'accouplement.

3. Gain net de gestation : différence entre le poids de la truie après la parturition (expulsion du placenta) et le poids à l'accouplement.

4. Perte de poids à la parturition : différence entre le gain brut et le gain net de gestation.

5. Poids de la portée à la naissance : somme des poids des porcelets nés vivants et des porcelets mort-nés.

6. Poids du placenta.

7. Pertes liquides à la parturition : différence entre la perte de poids à la parturition et la somme des poids du placenta et de la portée.

8. Perte de poids de lactation : différence entre le poids de la truic après sevrage $(60 \mathrm{j})$ et le poids après la parturition.

8 bis. Bilan gestation-lactation : différence entre le poids de la truie au sevrage et le poids à l'accouplement. parturition.

9. Effectif de la portée à la naissance : nombre total des porcelets vivants et mort-nés à la

Io. Nombre de porcelets viables : nombre des porcelets vivants 48 heures après la parturition.

II. Effectif de la portée au sevrage. vrage.

12. Pertes totales en porcelets : différence entre l'effectif de la portée à la naissance et au se-

I3. Poids de la portée à 2 I jours : somme des poids des porcelets à 2 I jours.

14. Poids de la portée au sevrage : somme des poids des porcelets à 60 jours. sance.

15. Poids moyen des porcelets à la naissance : poids de la portée divisé par l'effectif à la nais-

I6. Poids moyen des porcelets au sevrage : poids de la portée au sevrage divisé par le nombre de porcelets sevrés. 


\section{Méthode statistique}

On a tout d'abord calculé pour chaque classe (numéro de portée) en utilisant toutes les données disponibles, la valeur moyenne et l'intervalle de confiance de chacune des 16 variables (a l'exception du bilan gestation-lactation dont la détermination a été faite d'après les moyennes générales). Ces moyennes ont été ensuite comparées, après classement en série ordonnée croissante, selon la méthode de Duncan (1955) étendue au cas d'effectifs inégaux (Kramer, 1956; ArNaUd, 1964).

Compte tenu des effets du numéro de portée et de l'année de naissance (AUMAITRE et al., I966), les données ont ensuite été réparties en 30 sous-classes (numéro de portée, année de naissance) à l'intérieur desquelles nous avons calculé les corrélations linéaires entre les différentes variables.

\section{RÉSULTATS}

\section{A - Valeurs moyennes}

Les tableaux I et I bis rapportent, pour chacune des variables, les valeurs moyennes calculées sur l'ensemble des données (colonne I), ou après classement par numéro de portée. Ces valeurs sont accompagnées de leur intervalle de confiance

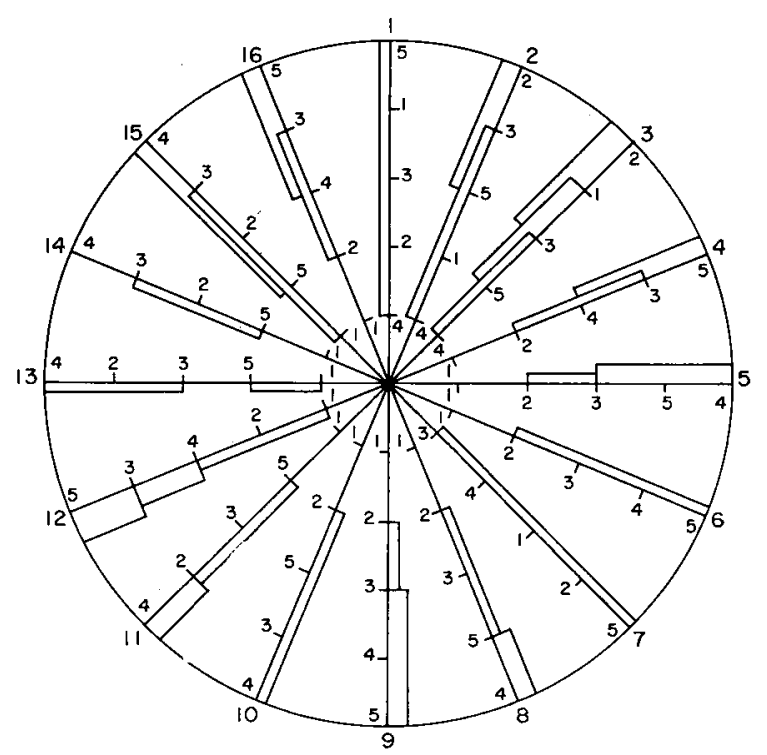

FIs. I. - Evolution de 16 caractéristiques de la portée en jonction de son numéro

Résultats du test de comparaison des moyennes ordonnées (1)
r. Durée de gestation
2. Gain brut gestation
3. Gain net gestation
4. Perte parturition
5. Poids total portée naissance
6. Poids placenta
7. Pertes liquides parturition
8. Perte de poids lactation
9. Effectif total naissance
10. Effectif viable naissance
I . Effectif $60 \mathrm{j}$
I2. Pertes totales porcelets
I3. Poids portée $2 \mathrm{I}$ j
I4. Poids portée $60 \mathrm{j}$
I5. Poids moyen naissance
I6. Poids moyen 60 j

(1) Pour chacune des 16 variables, les moyennes correspondant à 5 numéros de portée ont été répar$t$ ies en valeur croissante sur les i 6 rayons d'un cercle (ordre centrifuge); puis, les groupes de movennes q!i ne diffèrent pas significativement ont été réunies par un trait continu (DunCAN, I955). 


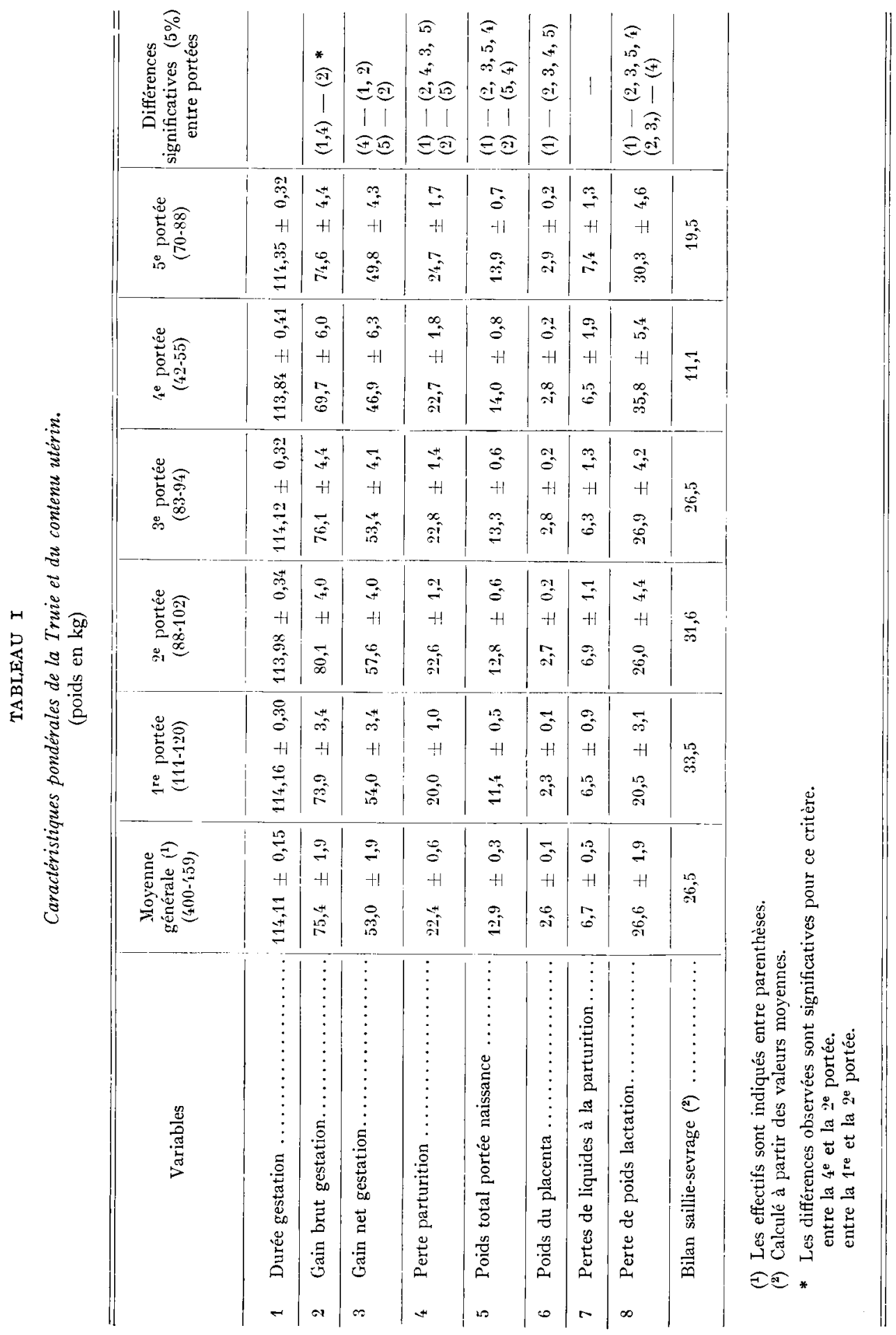


VARIATIONS PONDÉRALES DE LA TRUIE

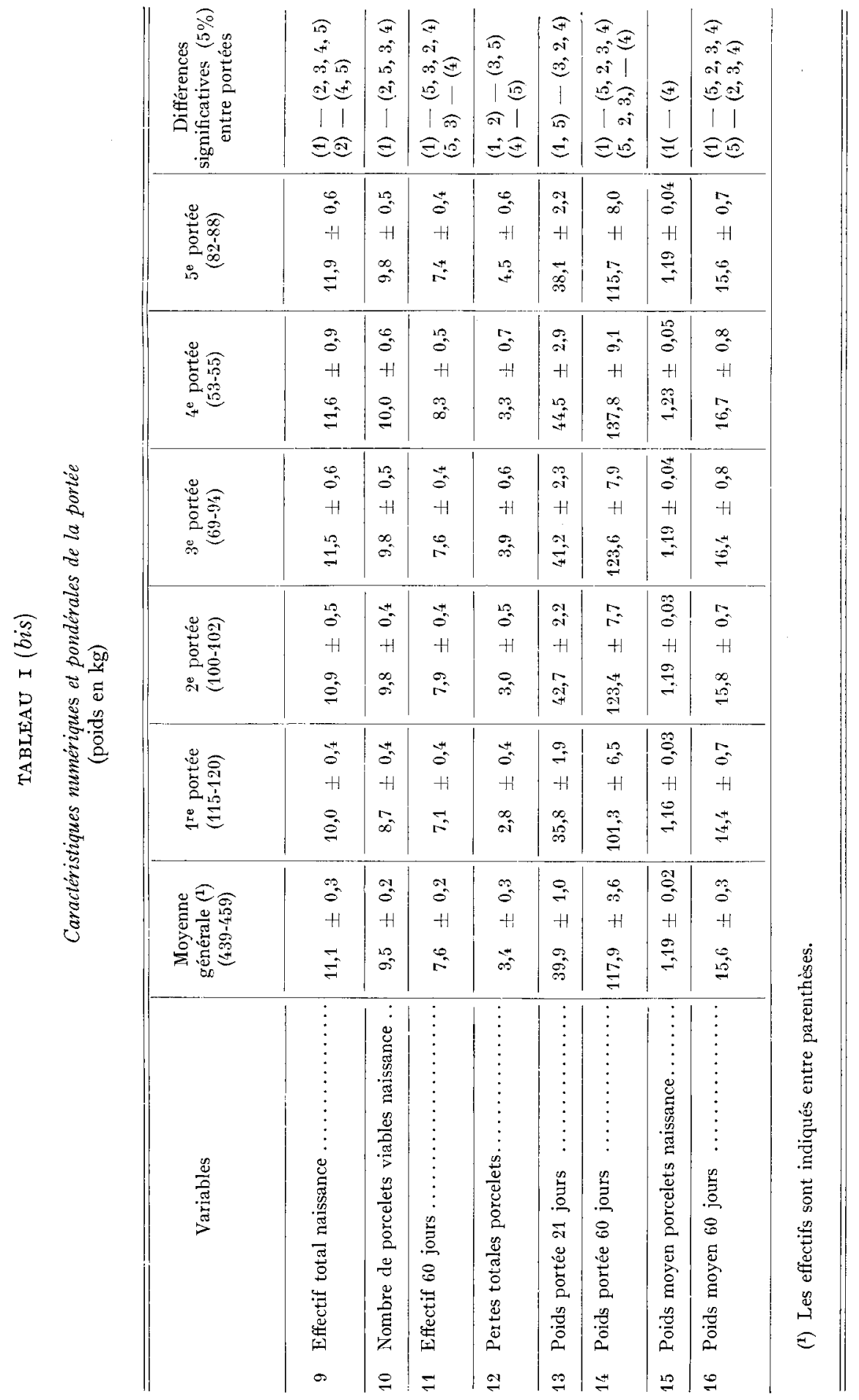


à $5 \mathrm{p}$. roo $\left( \pm 2 s_{\vec{x}}\right)(\mathbf{1})$ et la dernière colonne indique les différences significatives. Nous donnons en outre à la figure I une représentation graphique de la signification de ces différences.

On remarque que, lorsqu'elles existent, ces différences sont assez importantes entre la première et la seconde portée, mais beaucoup plus faibles ensuite. C'est le cas de la plupart des variables, à l'exception des pertes totales en porcelets (plus importantes chez les truies âgées) et du bilan gestation-lactation (plus faible chez les truies âgées).

La plupart des valeurs passent par un maximum, les unes au cours des premières portées (gain brut, gain net, perte de poids lactation), les autres à un stade plus tardif (effectif et poids des porcelets aux différents âges). Quelques-unes évoluent dans le même sens tout au long des 5 portées considérées (effectif total à la naissance, pertes totales en porcelets, poids du placenta, bilan saillie - sevrage). D'autres évoluent peu (poids moyen des porcelets à la naissance, effectif à 60 jours). Les variations de ces différents caractères ne sont donc pas toujours simultanées, ce qui peut être l'indice d'un certain décalage dans la chronologie des fonctions (croissance, reproduction, lactation).

\section{$\mathrm{B}$ - Évolution du poids vif des truies}

Bien que n'ayant pas fait l'objet d'une analyse systématique dans le cadre de cette étude, l'évolution du poids vif des truies aux différentes étapes de la reproduction est intéressante à considérer (LODGE et al., I96I). Nous en donnons la représentation, pour une période allant de la $\mathrm{I}^{\mathrm{re}}$ à la $9^{\mathrm{e}}$ portée, dans la figure 2 . Aux

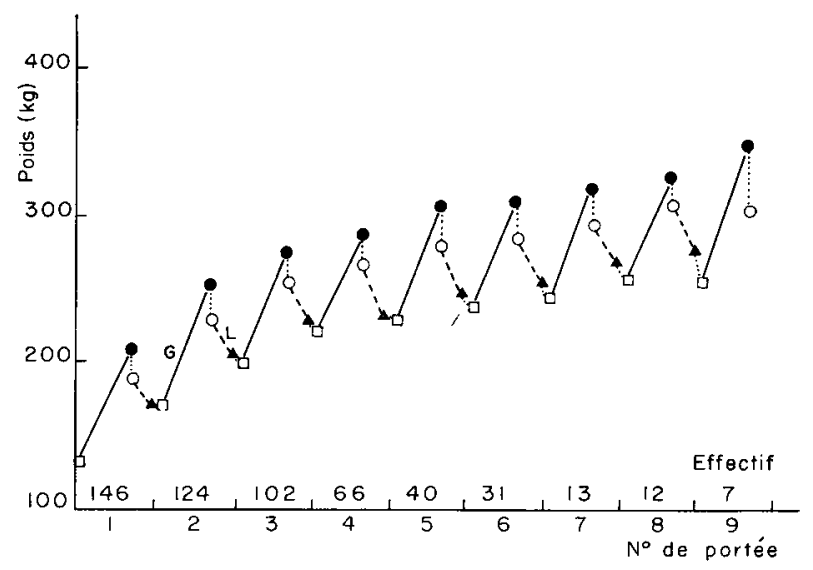

FIG. 2. - Évolution du poids moyen des truies au cours des différents cycles de reproduction

ㅁ Poids à la saillie

- Poids avant mise bas

- Poids après mise bas

- Poids au sevrage

- Gestation

-- Lactation

(1) $s_{x}$ : écart type de la moyenne. 
variations près de chaque cycle, le poids augmente d'une façon continue, mais dégressive au cours des portées successives. Par ailleurs, cette évolution est sensiblement la même, que l'on considère le poids à la saillie, à la parturition ou au sevrage.

\section{C - Répartition du gain de gestation}

La figure 3 présente l'évolution, de la Ire portée aux suivantes, de la répartition des différents éléments constitutifs des variations pondérales en cours de reproduc-
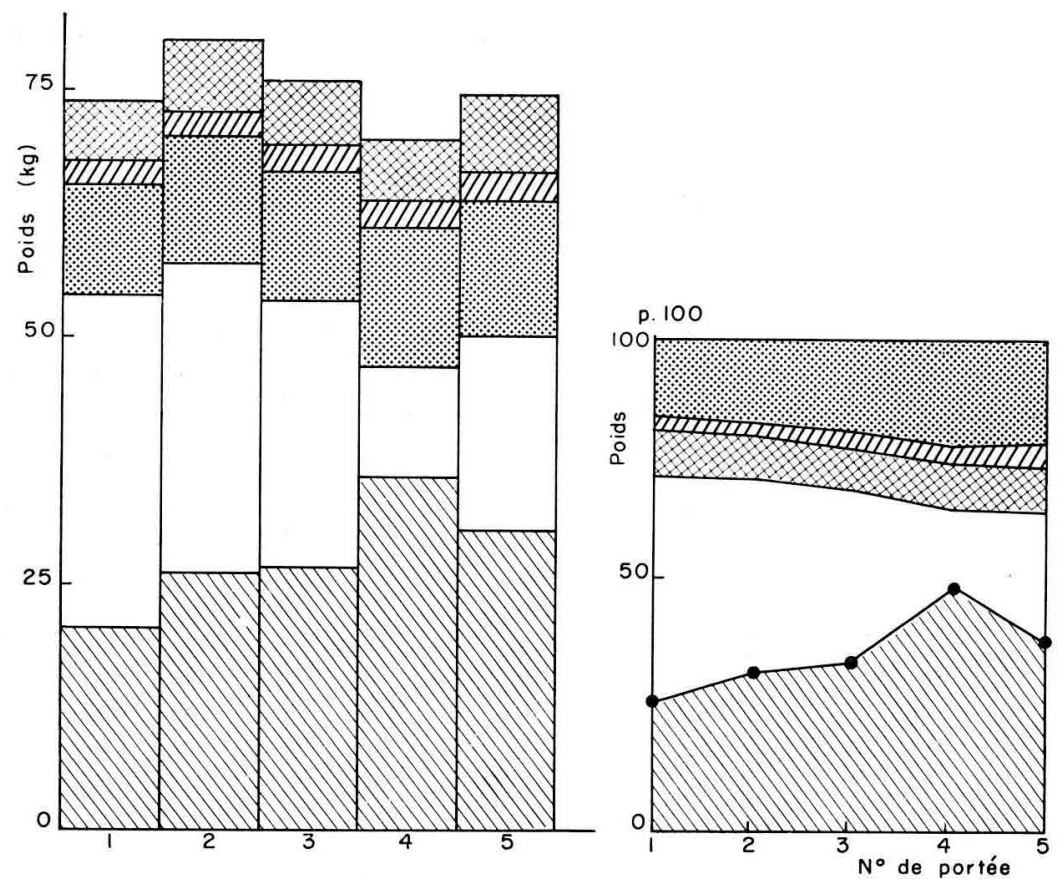

FIG. 3. - Répantition des gains et pertes de poids au cours de la gestation el de la lactation (Valeur absolue, $\mathrm{kg}$ et valeur relative, p. roo).

\begin{tabular}{ll}
\hline & Bilan gestation-lactation \\
Pertes de poids à la parturition \\
PDIDID & Poids du placenta \\
& Poids de la portée à la naissance \\
& Pertes de poids de lactation
\end{tabular}

tion. Les résultats sont exprimés en valeur absolue et en valeur relative (p. Ioo du gain brut). Ils concernent : le gain net de gestation (ou bénéfice maternel), le poids du contenu utérin (porcelets et placenta) et les pertes liquides à la parturition. - Cellesci comprennent les liquides utérins (amniotique et allantoïdien), de la vapeur d'eau 
que la truie élimine en grande quantité pendant le part (perspiration) et, éventuellement, de l'urine.

Nous avons également fait figurer sur le tableau I les pertes de lactation qui, par déduction, permettent de déterminer le gain net du cycle somplet (bilan gestation -- lactation).

Quel que soit le numéro de portée, l'importance relative des produits de la conception est faible (18-24 p. I0o) en regard du gain propre de la mère au cours de la gestation (65-72 p. 100). Mais cette répartition a tendance à se modifier légèrement au cours des portées successives : importance croissante du poids de la portée, diminution du gain net. Comme par ailleurs les pertes de lactation paraissent augmenter, il s'ensuit que le bilan du cycle complet diminue, ce qui apparaissait déjà dans l'évolution du poids vif (fig. 2).

\section{$\mathrm{D}-$ Corrélations}

L es valeurs des coefficients de corrélation intra-année et numéro de portée entre les $I 6$ variables considérées (le bilan saillie - sevrage ne figure pas dans ce calcul) sont rapportées au tableau 2 .

Bien qu'assez faibles dans l'ensemble, ces valeurs varient largement suivant les critères considérés. Un grand nombre de ces corrélations sont significatives, ce qui montre la variété des relations qui existent entre les différentes caractéristiques de la reproduction. L'interprétation toutefois doit être assez prudente pour deux raisons :

- Il s'agit des corrélations linéaires, par conséquent, l'absence de corrélation peut masquer une liaison curvilinéaire réelle.

- L'importance d'une corrélation totale peut résulter de la dépendance des variables. Ainsi, le gain brut et le net de gestation dépendent tous deux du poids à l'accouplement : toute erreur sur cette mesure entraîne une augmentation de la corrélation entre les deux variables. Toute corrélation entre variables dont la détermination est fonction de mesures communes peuvent ainsi être systématiquement augmentées ou diminuées.

Nous avons réparti arbitrairement les résultats précédents en trois groupes d'intérêt différent :

- Coefficients de corrélation élevés $(r>0,60)$. On trouve parmi eux ceux qui relient deux mesures d'un même critère pris à des époques différentes ou sous deux expressions différentes :

Gain brut/gain net de gestation $\ldots \ldots \ldots \ldots \ldots \ldots \ldots \ldots+0,94$

Nombre de porcelets viables/effectif sevrage $\ldots \ldots \ldots \ldots+0,66$

Poids de la portée à $2 \mathrm{I}$ jours/poids de la portée à $60 \mathrm{j} \ldots+\quad+0,80$

Effectif à la naissance/poids de la portée naissance ...... + $+0,79$

Effectif à $60 \mathrm{j} /$ poids de la portée à $60 \mathrm{j} \ldots \ldots \ldots \ldots \ldots+0,78$

Ces relations présentent l'intérêt de préciser numériquement l'importance de certains faits.

- Coefficient de corrélation de valeur moyenne $(0,20<r<0,60)$. Ils constituent la majorité des cas. Parmi eux, se trouvent notamment ceux qui relient 


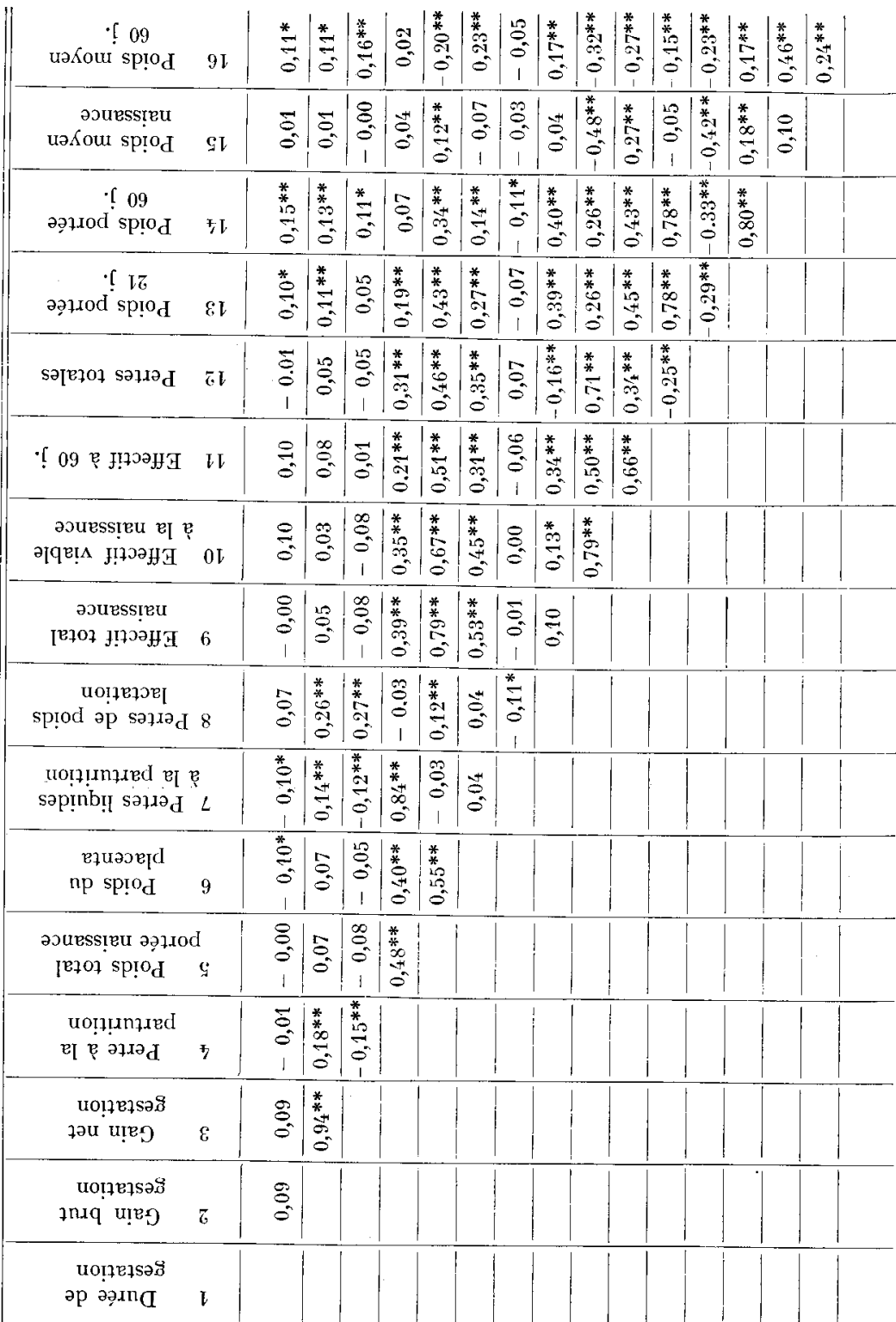


un caractère " Truie " (variation de poids) à un caractère "Porcelet " (effectif ou poids des portées). Ce sont donc les plus intéressants :

Gain brut (net) de gestation/perte de lactation ........

Perte de poids lactation/effectif porcelets viables ........ $+0,34$

Perte de poids lactation/poids portée $60 \mathrm{j} \ldots \ldots \ldots \ldots \ldots+0,40$

Poids du placenta/perte de parturition ..............

Mortalité $0-60 \mathrm{j}$ poids moyen naissance $\ldots \ldots \ldots \ldots \ldots \ldots \ldots, 0,42$

sevrage $\ldots \ldots \ldots \ldots \ldots \ldots-0,27$

- Coefficients de corrélation de valeur faible ou nulle $(r<0,20)$. Lorsque la valeur est nulle, elle peut signifier l'absence de liaison linéaire, ce qui était imprévisible dans certains cas :

Durée gestation/poids (ou effectif) portée naissance

Gain brut (net) gestation/poids moyen naissance

Effectif (poids) portée naissance/pertes liquides

Par contre, il existe une corrélation faible mais significative qui permet de soupçonner une liaison entre les caractères :

Gain brut (net) gestation/poids portée $60 \mathrm{j} \ldots \ldots \ldots \ldots+0, \mathrm{I} 6$

Perte poids lactation/mortalité à $60 \mathrm{j} \ldots \ldots \ldots \ldots \ldots \ldots \ldots \ldots$

Durée gestation/poids portée $60 \mathrm{j} \ldots \ldots \ldots \ldots \ldots \ldots \ldots+0, \mathrm{I} 5$

Poids placenta/durée gestation $\ldots \ldots \ldots \ldots \ldots \ldots \ldots \ldots \ldots$, Io

Ces caractères sont très éloignés et la signification de telles relations n'est pas toujours claire.

\section{DISCUSSION}

Les résultats concernant les valeurs moyennes des différents critères et leurs variations avec l'âge des animaux (numéro de portée) s'accordent assez bien avec ceux déjà trouvés par d'autres auteurs dans des conditions analogues (MAJERciak, I962; SQuiers et al., I952; Schafer et Granz, I955; Bowman et Bowland, I96I ; LODGE et al., I96I). On vérifie notamment l'augmentation des gains net et brut de gestation et l'amélioration de la plupart des performances d'élevage entre la première et la deuxième portée. Mais nos résultats montrent, en outre, que cette évolution se poursuit à des degrés divers pour certains caractères au moins jusqu'à la $5^{\mathbf{e}}$ portée.

Même si les différences d'une portée à la suivante tendent à s'amenuiser (à 1'exception de la mortalité) nous devons remarquer un certain parallélisme entre les variations de poids de l'animal (poids, gain de gestation, perte de lactation) et les performances d'élevage (nombre et poids des porcelets). L'importance du poids vif des reproducteurs a été souvent évoquée chez le Porc (ZELLER et al., I937; Donal,D et Fleming, I938; SQUiERs et al., I952 ; SAlmon-LEGAGNeUr et al., I960; Smith, I960; Bowman et Bowland, I96I ; LodGe et al., Ig6I ; OMrTvedt et al., I965) et chez les bovins (BERGE, I955 ; Milier et MCGILLIARD, I958) : les animaux 
les plus lourds sont en général les plus productifs. On en vient naturellement à rechercher la nature de cette relation entre la croissance et la reproduction. Deux hypothèses sont possibles, soit qu'il s'agisse de variations concomitantes : dans ce cas, l'amélioration des performances d'élevage coïnciderait avec le fait que 1'animal termine sa croissance et acquiert sa maturité sexuelle. Mais ceci n'est pas vrai pour tous les caractères puisque le poids maximum de la portée est atteint à la $4^{\mathrm{e}}$ lactation alors que la croissance se poursuit longtemps encore (fig. 2) ; ou bien les performances d'élevage sont en relation directe (dépendance) avec les variations de poids. L'une des théories les plus couramment rencontrées par le passé était qu'un gain de poids élevé au cours de la gestation était l'indice d'une bonne fertilité ( $Z$ ELLER et al., I937) et qu'il permettait à la truie de mieux faire face aux besoins intenses dela lactation, d'où la pratique de la suralimentation à cette époque (MCKENZIE, Ig28 ; JESPERSEN, I949). Mais cette opinion est loin d'être unanime, certains auteurs pensant au contraire qu'une prise de poids importante ou un excès d'adiposité peut être préjudiciable au développement des embryons et au bon déroulement de la lactation (DAvidson, I948; Carrol, et Krider, I950; KRUTYPPOROKH et SALEJ, I957; MoUSTGAARD, I959).

La comparaison des critères concernant la gestation, la portée et la lactation (tabl. 2 et fig. 2) nous permet de prendre une position plus nuancée. On constate que, pas plus l'augmentation du gain net de gestation ( $2^{\mathrm{e}}$ portée) que sa diminution (portées suivantes) ne semblent avoir entravé l'augmentation de l'effectif ou du poids des portées. Par contre, l'augmentation des performances de lactation s'est accompagnée de plus grandes pertes de poids vif pendant cette période, ce qui redonne du crédit à la première hypothèse.

Mais c'est surtout l'étude des corrélations qui apporte les renseignements les plus précis, car elle permet de mieux dissocier 1'influence des différents facteurs intervenant, soit sur les variations de poids des truies, soit sur les performances d'élevage.

a) Les variations de la durée de gestation sont très faibles, en accord avec HaRrNG et GRUHN (I965) ; elles restent pratiquement sans effet, tant sur le gain de poids maternel, que sur les caractéristiques des portées. Ce fait peut paraître surprenant en ce qui concerne le bénéfice maternel et pourrait signifier que la croissance en fin de gestation se limite au développement du seul contenu utérin; I1 l'est moins en ce qui concerne les embryons, car il est connu que chez la plupart des mammifères multipares, la durée de gestation varie en fonction inverse du nombre de petits (Biggers et al., I963). Ce fait a d'ailleurs été vérifié chez le Porc par Perry (I956) et récemment par LyNCH (I965) et OMTVED'T et al. (I965). Mais cet effet est relativement peu important et Cox (I964) ne trouve pas non plus de corrélation entre la durée de gestation et le gain de poids des truies ou le nombre d'embryons. La corrélation que nous observons entre la durée de gestation et le poids de la portée à $2 \mathrm{I}$ jours ou au sevrage $(r=+0, \mathrm{I} 5)$ reste, par contre, inexpliquée.

b) Le gain brut de gestation représente, dans la proportion de $80 \mathrm{p}$. Ioo, le gain net de la mère. Il est donc normal que ces deux valeurs soient très étroitement liées $(r=+0,94)$. Mais il est plus étonnant de constater que le contenu utérin (pertes à la parturition) n'influence que très faiblement le gain brut et que ce dernier est pratiquement indépendant du nombre et du poids des embryons. 
Trois conséquences en découlent :

- On ne peut préjuger de la fertilité ou de la prolificité d'une truie (nombre d'embryons) d'après son seul gain de poids ou son embonpoint extérieur.

- Un niveau d'alimentation élevé, qui passe pour stimuler fortement le gain de poids de gestation chez la Truie (SALMON-LEGAGNEUR et al., I960) n'a sans doute que peu d'effet sur la croissance embryonnaire.

- On ne peut se baser sur le poids ou le gain de poids de la mère pour évaluer le besoin de gestation pris au sens strict (contenu utérin).

c) La signification du gain net de gestation est la même que celle du gain brut à quelques nuances près. Le calcul du gain net par déduction des autres gains ou pertes de gestation est à l'origine de la corrélation négative entre ces deux valeurs. OMTVEDT et al. (I965) avait aussi trouvé une corrélation faiblement négative entre le gain net et le nombre de porcelets produits. On peut toutefois proposer une autre explication à ce fait, c'est que la mère et les embryons se comportent en concurrents dans la répartition des ressources alimentaires. En fait, on sait maintenant que l'embryon est toujours prioritaire et que la mère ne bénéficie que des reliquats laissés disponibles par ce dernier (SAlmon-LEGAGNEuR, I965). Il n'y a donc pas lieu de penser, comme le proposent certains, que l'adiposité ou l'embonpoint des truies est à l'origine de la baisse de fertilité ou de prolificité qui l'accompagne parfois (MoustGAARD, I958). Le contraire serait plus vraisemblable.

Mais quelle que soit l'explication véritable, on retiendra avec certitude qu'une augmentation du gain net de gestation, c'est-à-dire en définitive du niveau nutritionnel, est en faible corrélation avec les caractéristiques de la portée à la parturition.

d) I.es pertes à la parturition sont constituées essentiellement par le contenu utérin et accessoirement par les pertes corporelles liquides de la truie (perspiration, urine). Paradoxalement, ce sont ces dernières qui varient le plus puisqu'elles influencent davantage la perte totale, que ne le font le poids ou le nombre des embryons. Cela peut provenir en partie des erreurs d'estimation qui sont plus grandes pour les pertes appréciées par différences. Mais il est intéressant de noter que ces pertes liquides sont en relation positive avec le gain de poids brut de gestation $(r=+0, \mathrm{I} 4)$, ce qui semble indiquer la nature œémateuse d'une partie de ce gain de gestation. On peut ainsi penser, comme le suggèrent certains praticiens, que les truies qui ont effectué des gains de poids élevés éprouvent davantage de difficultés atı moment de la parturition. L'augmentation de la dépense physique due à cet acte entraînerait une perte énergétique, accompagnée d'une déshydratation, plus importante. Il ne semble pas qu'on puisse accorder d'autres significations à ces pertes à la parturition en raison d'une liaison assez élevée et inexpliquée avec le poids du placenta et le nombre de porcelets.

e) Les caractéristiques des portées à la naissance (poids, nombre des embryons, importance du placenta) et les relations qui les unissent sont bien connues (DUNCAN et LODGE, I96I). Il nous suffit de constater une nouvelle fois que le poids moyen des porcelets est fonction de l'effectif des portées $(r=-0,48)$ et qu'il intervient non seulement sur le poids moyen au sevrage $(r=+0,24)$, mais aussi sur la mortalité au cours de l'allaitement $(r=-0,42)$. Ceci conduit à souligner avec de nombreux auteurs (KoRkMAN, I947; CESBRON, I958; OMTVEDT et al., I965) l'intérêt 
de surveiller très attentivement tous les facteurs qui conditionnent le poids à la naissance.

f) Les pertes de poids de la Truie en lactation semblent, enfin, présenter une signification bien précise. Le fait qu'elles soient en corrélation avec le poids et le nombre des porcelets à différents âges, mais plus particulièrement au sevrage $(r=$ $+0,40)$, indique clairement la part qu'elles jouent dans la performance d'allaitement. C'est bien en effet en tant que substances de réserves aisément mobilisables qu'elles interviennent pour compléter les ressources nutritionnelles et notamment 1'approvisionnement en nutriments énergétiques chez la Truie (SAI.MON-LEGagneur, I965) et chez la Vache laitière (DECAEN et JoUnNET, I966). Ce transfert est rendu possible grâce à la priorité absolue exercée par les besoins de lactation sur les autres fonctions de la Truie. Il en résulte que les truies les plus laitières sont celles qui perdent le plus de poids (ZELLER, et al, I937 ; SCHAFER, et GrANZ, I955; SALMON-LEGAGNEUR, I958; BoWMAN et BowlAND, I96I), ce qui a contribué parfois à accréditer l'opinion hâtive qu'une bonne truie devrait être maigre. On peut penser au contraire que ce phénomène traduit davantage l'insuffisance de l'apport alimentaire, puisque presque tous les auteurs, à l'exception de SCHAFER et GRANz (I955), sont d'accord pour reconnaitre que la perte de poids est en relation avec le nombre de petits allaités (ZELLER, et $a l$., I 937 ; VANschoubroek, et Fimbo, I 962 ; MAJERciak, I 962 ; BowmaN et Bowland, I96r).

Il reste à connaître dans quelles conditions la truie en lactation peut perdre du poids. Cette perte de poids est systématique. Selon SchafER et Granz (I955), il y aurait une prédisposition génétique ; selon LENKEIT et al. (I956), le phénomène serait lié al1 catabolisme azoté obligatoire imposé par l'involution de l'appareil reproducteur qui apparaît même en l'absence de sécrétion lactée. S'il paraît plus logique de relier cette perte de poids à l'insuffisance de l'apport alimentaire de lactation (SALMON-LEGAGNEUR, I965), cette explication ne suffit pas toujours et VANSchouBROEK et ЕмBo (Ig62) croient davantage à un mécanisme hormonal. En tous cas, il y a une liaison assez étroite entre le gain de gestation et la perte de lactation $(r=+0,27)$. La Truie ne peut perdre du poids que dans la mesure où elle en a gagné pendant la gestation (DEAN, et 'Tribble, I960; SAlmon-LEGagNeUr, I965). Inversement, une truie qui a pris peu de poids en cours de gestation, peut gagner du poids au cours de la lactation (SALMON-LEGAGNEUR, I965).

En conclusion, la stimulation de la production laitière par l'intermédiaire des variations de poids ne peut se faire qu'au prix d'une surcharge alimentaire importante au cours de la gestation (STEVENSON, et EILIS, I957; SMith, ig6o $a$; SAlMON-LEGAGNEUR, et al., I960). Mais s'agit-il d'une technique valable sur le plan économique? On peut en douter. C'était déjà ce qu'avaient conclu Smirh (I960 b) et SALMON-LEGAGNEUR (I965) à l'issue de leurs expériences. C'est encore ce que confirme la faible corrélation entre le gain de poids net de gestation et la performance de lactation $(r=+0$, II à $+0, I 6)$ qui ne laisse espérer qu'une influence d'environ 2 p. Ioo sur la variation du poids de la portée au sevrage. 


\section{SUMMARY}

\section{RELATIONSHIP BETWEEN WEIGHT CHANGES IN THE BREEDING SOW AND THE EFFICIENCY OF PRODUCTION}

This investigation bears on 459 litters farrowed by 186 Large White sows bred in the Station de Recherches sur l'Élevage des Porcs between 1954 and $196 \mathrm{I}$.

The sows were weighed at the time of mating, before and after farrowing and at the time of weaning (6o days). The litters were weighed at birth and when 21 and 60 days old. On the whole, I6 variables concerning either weight variations in sows or litter characteristics were studied.

r. The means corresponding to the first five farrowings were compared by means of Duncan's multiple range test (table I and I $b$, fig. I). The weights of the sows increase during pregnancy $(45-57 \mathrm{~kg})$ and decrease during lactation $(20-35 \mathrm{~kg})$ so that the weight change during each repro. ductive cycle is positive (fig. 2), Litter size and weight at birth and at the time of weaning in. crease up to the 4 th farrowing, whereas piglet mortality reaches a maximum at the ${ }^{\text {th }}$ and subsequen ${ }_{t}$ litters. On another hand, the duration of pregnancy is not related to the serial number of the litter.

2. The linear correlation coefficients between the I6 variables were calculated within each year of birth and within the same serial number of litters. There is scarcely any relation between litter size and weight at birth and weight gain of the sow during pregnancy.

Weight gain during pregnancy and loss during lactation are correlated $(r=0.27)$; weight loss during lactation is correla ted with litter weight at $2 \mathrm{I}$ and 60 days $(r=0.40)$.

However, it appears that any increase in the gain during pregnancy does not considerably improve litter characteristics (correlation gestation gain to weight of the litter at the time of wea ning : $r=0 . \mathrm{I}$ ).

\section{RÉFÉRENCES BIBLIOGRAPHIQUES}

Arnavd A., 1964. Application d'un test de comparaison de deux moyennes d'une série ordonncée au classement des taureaux sur descendance. Ann. Zootech., 13, г73-18г.

Aumaitre A., Legault C., Saimon-Legagneur E., 1966. Aspects biométriques de la croissance pondérale du Porcelet. Ann. Zootcch. 15, (à paraître).

BERGE S., 1955. Body weight and milk production. Tidsskr. Norske Landbr., 62. 59-70.

Biggers J. D., Curnow R. N., FinN A., Ig63. Regulation of the gestation period in mice. J.Reprod. liert., 6, 125 .

Bowman G. H., Bowland J. I.., I96I. An appraisal of certain sources of environmental variation in the productivity of sows. Canad. J. nim. Sci., 41, 220-229.

Carrol W. E., Krider J. L., I950. Swine Production. Mc Graw Hill, New York.

Cesbron, ig58. Données non publiées.

Cox D. F., 1964. Relation of litter size and other factors to the duration of gestation in the pig. J. Reprod. Fiertil., $7,405-408$.

Davidson H. R., r948. Production and marketing of pig. Longmans green. London.

Dean B. T., Tribble L. F., r959. The effect of level of feeding during gestation on sow performance. Univ. Missouri, 3rd Sivine day, p. I 2.

Dean B. T., TRIBble I. F., I 960 . Effect of level of energy intake during gestati.n on condition and performance of swine (Abstr.). J. unim. Sci., 19, г257.

Decaen C., Journet M., ig66. Données non publiées.

Donald H. P., Fleming; I., 1938. Effect of prenatal weight-changes in breeding sows on the number and size of new born pigs. limp. J.exper. Agric., 6, 34:-3+9.

Duncan D. B., I955. Multiple range and multiple $F$ tests. Biom., 11, I-42.

Duncan D. L., Lodge G. A.. I961. Diet in relation to reproduc and the viability of the young. Part 3 . Pigs. Commonwealth Agric. Bureaux Tech. Comm. 21. Farnham Royal. I 5.

Haring F., Gruin R., I965. Untersuchungen zur Trächtigkeidauer beim Schwein. Schweinezucht u. Schweinm tst, I3, I $24-26$.

Jespersen J., I949. Normes d'alimentation du Porc. Rapp. part. Ve Congrès Zootechnie, Paris, p. 33. 
Korkman N., 1947. Causes of variation in the size and weight of litters from sows. Acla Agric. Suecana, 2-3, $253-310$.

KRAmer C. Y., I956. Extension of multiple range tests to group means with unequal numbers of replications. Biom., 12, 307-310.

KRutyporokh F. I., SALEJ P. I., I957. Influence of nutrition and environment on pregnant sows. Svinovodstvo, 6, 33-36.

Lauprecht E., SaAthoff. T., Watter E., I962. Zum Einfluss der Säugeleistung auf die Rückenspeckdicke von Sauen. Z. Tierz. ZuchtBiol., 76, 374-387.

Lenkeit W., Gutte J. O., Streuter-Petermoller A., i955. Langfristige Untersuchungen zum ausseren und inneren Stoffwechsel des graviden und laktierenden Schweines. 2. Der Ablauf der N-Bilanz vom Ende der Gravidität bis zum Ende der Laktation bei gleichbleibender Emährung. Z. Tierernährung Fullermiltelk, 10, 228-240.

Leroy A. M., 1949. Normes d'alimentation du Porc. Rapp. Gén. Ve Congrès Zootech., p. 23, Paris.

Lodge G. A., McDonald I., MacPherson R. M., 1961. Weight changes in sows during pregnancy and lactation. Anim. Prod., 3, 269-275.

Lynci G., 1965. A study of the reproductive characteristics of pigs. Meld. Norg. Landbr., 44 (9), p. 9.

McKenzie F. F., 1928. Growth and reproduction in swine. Agric. Exp. Sta. Missouri. Res. Bull., Ir8.

Majerciak P., I962. Étude de la variation du poids vif des truies durant la gestation et la lactation (Tchèque). Zivocisna Vyroba 35, 269-286.

Miller R. H., Mc Gilliard L. D., I958. Relation between weight at first calving and milk production during the first lactation. J. Dairy Sci. (Abstr.) 41, 747

MoustgaARD J., I959. Nutrition and reproduction on domestic animals. Reproduction in dom estics animals. Vrll II HH. Col. et Cupps Acad. Press. New-York.

Omtvedt J. T., Stanislaw C. M., Whatley J. A. J., 1965. Relationship of gestation length age and weight at breeding and gestation gain to sow productivity at farrowing. J. anim. Sci., 24, 53โ-535.

PERRY J. S., I956. Observations on reproduction in a pedigree herd of Large-White pigs. J. agric. Sci., 47, $332-340$.

Salmon-Legagneur E., I958. Observations sur la production laitière des truies. Ann. Zootech., 7, I43-I62.

SALMON-Legagneur E., 1965. Quelques aspects des relations nutritionnelles entre la gestation et la lactation chez la Truie. Thèse Doctorat; Univ. Paris.

Salmon-Legagneur F., Gomez G., Jacquot R., ig6o. Influence de la suralimentation en fin de gestation sur la production laitière de la Truie. C. R. Acad. Agric., Fr., 46, 445-450.

Scirafer H., Granz E., I955. Beobachtungen über die Gewichtsverluste der Sauen während der Säugezeit. Züchtungskunde, 27, 37-46.

Sмiтн D. M., I960 a. The effect of condition at farrowing upon the subsequent milk yield and the effciency of production. N. Z. J. Agric. Res., 598-616.

SмIтн D. M., I960 $b$. The yield and energy content of milk and the energetic efficiency of sows on different levels of nutrition during gestation and lactation. N.Z. J. Agric. Res., 3, 745-763.

Squiers C. D., Dickerson C. E., Mayer D. T., i952. Influence of inbreeding, age, growth rate of sows on sexual maturity, rate of ovulation and embryonic survival. Agric. Exp. Sia. Missouri, Res. Bull., 494.

Stevenson J. W., Ellis J. R., I957. Effect of gestation diets and creep feeding on livabiltiy and weight gains of suckling pigs. J. anim. Sci., 16, $877-885$.

VAnschoubroek F., Embo P., i 962 . Recherches concernant le volume, la cause et la nature de la diminution de poids chez les truies pendant la période de lactation (flamand). Vlaams diergeneeskd. Tijdschr., 31, I $29-144$.

Zeller J. H., Johnson T. C., Graft W. A., 1937. The significance of weight changes in sows during the gestation and suckling period. Amer. Soc. anim. Prod. Proc., p. i21-126. 